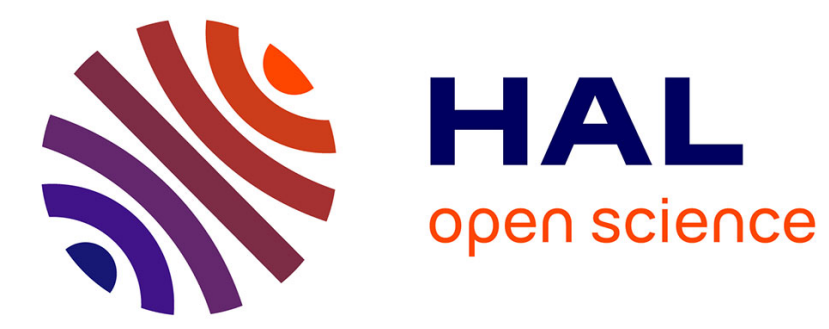

\title{
Le problème de la fidélité au réel dans la réalisation télévisée du football \\ Laurent Camus
}

\section{To cite this version:}

Laurent Camus. Le problème de la fidélité au réel dans la réalisation télévisée du football. Télévision, 2021, 12, pp.103-120. 10.3917/telev.012.0103 . halshs-03205602

\section{HAL Id: halshs-03205602 \\ https://shs.hal.science/halshs-03205602}

Submitted on 8 Jun 2021

HAL is a multi-disciplinary open access archive for the deposit and dissemination of scientific research documents, whether they are published or not. The documents may come from teaching and research institutions in France or abroad, or from public or private research centers.
L'archive ouverte pluridisciplinaire HAL, est destinée au dépôt et à la diffusion de documents scientifiques de niveau recherche, publiés ou non, émanant des établissements d'enseignement et de recherche français ou étrangers, des laboratoires publics ou privés. 
L. Camus (2021), « Le problème de la fidélité au réel dans la réalisation télévisée du football », Télévision, 12, p. 103-120.

\title{
Le problème de la fidélité au réel dans la réalisation télévisée du football
}

\author{
Laurent CAMUS
}

Université de Bâle, Département de Linguistique et Littérature

\begin{abstract}
Résumé :
L'article porte sur le problème de la fidélité au réel, exigence morale liant les médiateurs d'un événement à leur public, en prenant le cas de la réalisation télévisée du football en direct. Il relate d'abord les évolutions par lesquelles le match télévisé est passé du statut de captation transparente, dans lequel le lien à la réalité est non-problématique, à celui d'une mise en image dynamique interrogeant continument le statut des événements diffusés. Ces transformations de la médiatisation du football ont donné lieu à un certain nombre de critiques, visant en premier lieu les réalisateurs, accusés de reconstruire l'événement et de le rapprocher abusivement de la fiction. Après avoir interrogé ces critiques, l'article propose une voie alternative au blâme pour questionner la moralité des pratiques de représentation télévisuelle en direct. Par la prise en compte empirique du contexte local et des impératifs pratiques dans lequel les techniciens sont placés pour mettre en image la réalité, il est possible d'envisager le sens moral et les efforts dont ils font preuve pour rendre compte de la réalité telle qu'ils la perçoivent et pour en préserver l'intelligibilité pour le public distant.
\end{abstract}

\section{Introduction}

Il y a une attente tacite et ordinaire du téléspectateur regardant un événement sportif diffusé en direct à la télévision. Celle-ci porte sur le fait que le document visuel soit une représentation fidèle de la réalité de l'événement tel qu'il se déroule in situ. Cette attente, constitutive de l'expérience télévisuelle du sport, a une dimension contraignante pour le diffuseur et l'équipe de 
réalisation qu'il embauche. Par leurs actions, le réalisateur et les différents techniciens tentent de s'y conformer lorsqu'ils produisent les images par lesquelles ils rendent compte du match.

Relativement singulière à la télévision, l'attente de fidélité au réel est tellement considérée comme ordinaire et allant de soi lorsqu'on l'applique au sport qu'elle est longtemps passée inaperçue. Réalisé en temps réel - c'est-à-dire monté simultanément au déroulement de l'action et n'offrant donc pas la possibilité d'une reconstruction ex post-le match de football télévisé a longtemps entretenu un rapport non-problématique avec les faits dont il rend compte. Filmé en plan large avec un minimum de changements de plan, le match télévisé a été conçu comme une captation transparente vis-à-vis des faits. L'évolution des techniques, le renouvellement du paysage télévisuel et des liens institutionnels entre télévision et sport à partir des années 1980 contribuent à reconfigurer les modalités pratiques de mises en images du match. Le dispositif et les procédures visuelles inhérents à la retransmission gagnent peu à peu en visibilité, troublant en cela le rapport traditionnel à la réalité : la télévision apparaît non plus simplement comme une lucarne transparente sur l'événement mais aussi comme une instance contribuant réflexivement à en interroger et en révéler sa réalité.

Cet article vise à montrer que la règle morale de fidélité aux faits est accomplie de façon située et contextuelle par les techniciens. Son accomplissement ne doit pas être simplement jugé au vu des documents télévisuels finis mais doit être rapporté aux procédures locales et interactionnelles par lesquelles les techniciens perçoivent et mettent en images le match depuis la régie. Par leurs interactions, à la fois verbales et non-verbales (mouvements de caméras, sélection de plan et de ralentis), ils rendent ainsi visibles une certaine compréhension de l'événement et de sa réalité. L'article, inspiré de l'ethnométhodologie (Garfinkel, 1967), propose donc une approche praxéologique (Quéré, 1991) et située de ces pratiques réflexives par lesquelles les acteurs de l'audiovisuel font sens de la réalité qu'ils perçoivent, conception mettant en avant le caractère endogène de la moralité ${ }^{1}$.

Dans un premier temps, on présentera les évolutions des manières de réaliser les matchs de football à la télévision. On verra notamment comment s'est opéré le passage d'une conception de la mise en images comme captation transparente d'une réalité brute à une conception plus réflexive, caractérisée par des effets de montage plus marqués, dans laquelle la réalité du match

\footnotetext{
${ }^{1}$ Voir Turowetz \& Maynard (2010) pour une introduction à cette conception de la moralité.
} 
n'est pas considérée comme un donné mais comme un accomplissement télévisuel, comme le fruit d'un travail d'enquête par l'image.

Une deuxième partie de l'article reviendra sur une série de critiques portant sur ces nouvelles modalités de réalisation télévisuelle des matchs de football. Ces critiques mettent notamment en évidence l'existence d'une dimension morale dans les pratiques de mise en images et l'exigence, assignée aux réalisateurs, de représenter fidèlement la rencontre pour le public distant pour lequel la télévision constitue le seul moyen d'accès. Se pose ainsi la question de ce que la fidélité à la réalité veut dire. On verra qu'une telle position, qualifiée de constructiviste, pose de ce point de vue des problèmes théoriques et méthodologiques liés à la posture d'enquête et à la conception de la moralité engagées dans l'analyse.

Enfin, l'article proposera une conception alternative des programmes réalisés en direct et des règles morales qui leur sont afférentes. Contrairement à la conception constructiviste critique, on pourra qualifier cette conception d'endogène en ce sens qu'elle tentera de replacer les exigences morales inhérentes à la réalisation du football dans leur foyer d'émergence pratique. Par la prise en compte des interactions en régie, il est possible de montrer comment les techniciens de l'image se rapportent à la réalité qu'ils perçoivent à travers des écrans et mobilisent leur connaissance de sens commun pour le mettre en images.

\section{Les évolutions des modes de représentation du football télévisé et l'émer- gence du problème de la fidélité au réel}

\section{De la communauté de soutien à la communauté experte}

Traditionnellement vécu dans l'espace physique du stade, le match de football s'est progressivement transformé en un événement médiatique per se, dont la perception à distance, devenue la norme de la participation à l'événement, en a peu à peu transformé les modalités d'accomplissement. Jusqu'aux années 1980, la retransmission des matchs de football en direct à la télévision est ponctuelle. Diffusés sur des grandes chaînes publiques, ils donnent à voir à l'ensemble de la communauté nationale des téléspectateurs le match d'une équipe dont ils partagent l'identité nationale. Ce sont uniquement les matchs de coupe d'Europe ou de coupe du monde 
qui sont diffusés, voyant l'équipe de France ou parfois des clubs français (Saint-Etienne et Bastia notamment) affronter des équipes étrangères.

Le match de football télévisé est alors conçu sous une modalité agonistique et relève de ce que Dayan et Katz (1992) ont décrit dans les termes d'une « cérémonie télévisée », occasionnant la réunion de la communauté autour de cet événement. S'il existe bien une dimension morale à la diffusion de la rencontre, elle est alors à trouver dans le commentaire qui, dans un registre passionnel, subjectif et parfois outrancier, rend manifeste l'appartenance des médiateurs et de l'ensemble des téléspectateurs à une communauté réunie le temps du match autour du soutien à l'une des équipes. Cette communauté d'appartenance est actualisée à l'antenne sous la forme d'une paire catégorielle « nous-eux ».

Outre ce rapport engagé entretenu avec l'événement, l'une des caractéristiques de cette période est la conception de son déroulement comme étant autonome vis-à-vis de sa production médiatique. Les débuts de match ne sont pas toujours diffusés et les prises d'antenne tardives en raison de l'agenda spécifique de la chaîne ne sont pas rares. La réalisation visuelle du match est alors secondaire dans l'éditorialisation de la rencontre. Elle est conçue comme une simple captation transparente et surplombante du déroulement de l'action (parfois rapprochée de la vidéo-surveillance (Bideaux, 2007)) privilégiant un plan large unique pour rendre compte de ce qui se passe sur le terrain. Les changements de plan sont assez peu fréquents et les dispositifs filmiques relativement légers puisque, jusqu'au début des années 1980, on utilise rarement plus de trois caméras pour filmer un match. Les ralentis qui sont insérés sont en général des copies des plans diffusés en direct, en ce qu'ils redonnent à voir le même plan en décélérant les images. L'accès aux images archivées que sont les ralentis s'obtient par le rembobinage de machines analogiques qui rendent nécessaire un temps d'accès relativement important pour revenir au phénomène recherché.

Les années 1980 marquent un tournant dans les manières de mettre en images le football en Europe, en lien notamment avec la reconfiguration du paysage télévisuel. Avec l'apparition de chaînes privées payantes voyant dans le football une façon de proposer du contenu exclusif à bas coût, les championnats nationaux, jusqu'alors en large partie absents des écrans, font l'objet d'une diffusion exclusive. Le football télévisé devient dès lors un rendez-vous régulier et sériel et non plus simplement un événement ponctuel, limité aux grandes compétitions internationales.

Ces évolutions de la médiatisation du football sont particulièrement perceptibles en France, où Canal + entame en 1984 la diffusion du championnat de première division. La chaîne cryptée 
met ainsi en images une compétition jusque-là uniquement accessible depuis l'espace du stade et donc invisible pour le grand public. Par la retransmission de ces matchs opposant des clubs d'un même pays, la modalité de participation traditionnelle basée sur l'agôn ${ }^{2}$ et le soutien passionnel s'efface, pour laisser place à d'autres manières de se rapporter à la rencontre. Les matchs ne sont plus des événements exceptionnels réunissant ponctuellement une «communauté imaginée ", mais des événements récurrents, insérés dans la suite sérielle du championnat, réunissant la communauté réelle des abonnés. Contrairement à la première, celle-ci ne se caractérise pas par le soutien partagé à une seule et même équipe, mais au paiement d'un abonnement lui donnant accès à la retransmission des rencontres. Si l'abonné peut occasionnellement vivre le match en tant que supporter, il assiste le plus souvent à des rencontres opposant des équipes qui lui sont étrangères et vis-à-vis desquelles il peut n'éprouver aucun sentiment d'appartenance. Le match de football clivant laisse la place à des manières alternatives de se rapporter au jeu et à l'action aptes à rendre possible la participation de tous à l'événement et donc à garantir l'unité de la communauté des abonnés.

C'est ainsi qu'apparaissent des modalités expertes de se rapporter au jeu et aux actions accomplies par les joueurs (Papa, 1998 : 288-291). Elles permettent de vivre l'événement non pas simplement dans un rapport émotionnel mais dans un rapport de connaissance, d'expertise et de compréhension du jeu, dans ses dimensions techniques et tactiques, et dans son lien avec la règle. Ces nouvelles formes de médiatisation du football, à rapprocher du traitement journalistique ordinaire réclamant neutralité et symétrie, sont d'abord perceptibles dans le commentaire. Sa figure experte se caractérise en particulier par un rapport à l'événement à la fois plus distancié émotionnellement (les formes traditionnelles de soutien étant proscrites) et plus proche de l'action, qu'elle se déroule sur le terrain (avec une plus grande technicité des analyses, basée sur l'usage de statistiques) et ou dans les coulisses (avec des nombreuses interviews et indiscrétions en bord de terrain et dans les couloirs).

\footnotetext{
${ }^{2}$ L'agôn, c'est-à-dire la compétition, catégorie à l'intérieur de laquelle les rencontres de sport collectif sont classiquement rangées, que ce soit chez Caillois (1967 : 50-51) ou chez Dayan et Katz (1992:1) qui reprennent explicitement sa définition de l'agôn pour forger leur notion de contest (1992: 33, traduit par « confrontation » dans l'édition française).
} 
paru dans Télévision, 12, p. 103-120

\section{L'avènement d'une conception documentaire de la réalisation télévisée du football}

Une évolution majeure de la narration du football, centrale pour le propos développé ici, tient au fait que celle-ci s'adosse désormais à la réalisation, dont le rôle dans la médiatisation de l'événement est considérablement étendu. La production télévisuelle du match n'est plus conçue comme sa simple captation transparente. Un pouvoir nouveau est ainsi acquis par les réalisateurs. Ceux-ci ne sont plus de simples exécutants passifs d'une captation surplombante du match par un plan large unique. Les dispositifs de caméras s'étoffent et se complexifient, la mise en images devient dynamique, le montage et les mouvements de caméras rendant compte des trajectoires des actions de façon détaillée et accélérée.

Cette nouvelle conception de la réalisation, qu'on peut qualifier de « documentaire » (Bideaux, 2007 : 604), est notamment portée par deux réalisateurs : Jean-Paul Jaud (sur Canal+) et François-Charles Bideaux (sur TF1 puis Canal+). L'un et l'autre ont contribué à repenser entièrement les manières de rendre compte du match, à travers une réflexion sur le positionnement des caméras, le montage et l'usage des ralentis, de façon à faire du match de football télévisé un récit filmique du réel. Cette conception documentaire, perçue comme une forme d'accomplissement d'un cinéma du réel, tire profit de la singularité des événements filmés en direct et du pouvoir qu'elle donne au réalisateur (cf. Tesson, 2007). Car, si le document visuel qui est produit est contraint par l'événement - et l'impératif moral d'en préserver l'intelligibilité pour le public -, les réalisateurs de sport sont affranchis des contraintes classiques pesant sur la production audiovisuelle. Les spécificités du direct leur confèrent une mainmise totale sur le montage, aucune post-production ne venant altérer ou modifier leur travail.

Cette conception documentaire de la réalisation se caractérise par une alternance de plans larges, rendant visible l'ensemble des interactions sur le terrain, et de plans serrés montrant une partie de la réalité du stade, parfois extérieure au déroulement de l'action sur le terrain. Les plans serrés des joueurs sont mobilisés pour montrer les gestes techniques au plus près, ainsi que les émotions exprimées suite à des faits de jeu (joie, colère, douleur, etc.). L'alternance est à la fois une ressource pour documenter l'action au plus près et une procédure accélérant considérablement le montage et dynamisant le programme.

Outre l'alternance des plans de direct, les ralentis sont mobilisés de façon régulière, systématique et accélérée. Le flux de chacune des caméras est continuellement enregistré sur des plateformes et perçus par des opérateurs qui indexent en temps réel chacun des phénomènes, 
les stockent (de façon à produire notamment des résumés de match de façon rapide) et les proposent au réalisateur lors des pauses du jeu. Ainsi lors de chaque pause du match (faute, touche, etc.), des ralentis, montrant l'action sous une diversité d'angles ou de valeurs de plan, peuvent être diffusés à l'antenne. Les ralentis permettent de revoir l'action sous une multiplicité d'angles, objectivant par l'image le phénomène montré.

Le réalisateur joue un rôle fondamental de coordination dans ces nouveaux dispositifs de mise en image. Il est en charge de l'ensemble du dispositif et sélectionne les plans en temps réel. Il instruit le travail des cadreurs, leur placement et déplacements dans l'espace (plan de caméra des caméras fixe et déplacement des caméras mobile), les objets sur lequel ils placent la focale de leur caméra (joueur, arbitre, spectateur, ballon, etc.) et la valeur de plan (telle partie du terrain ou corps d'un joueur filmé ; de façon plus ou moins large).

Ces évolutions des manières de rendre compte du match s'adossent sur la croissance des moyens techniques et financiers mis à disposition des réalisateurs (de 5 caméras et 2 magnétoscopes en 1984, date de la diffusion de la première rencontre sur Canal+, à environ 25 caméras et 12 plateformes numériques de ralenti aujourd'hui). Celles-ci accompagnent également une série d'innovations techniques dans le domaine audiovisuel : l'amélioration des résolutions des caméras qui permet d'accéder à des détails des actions; la miniaturisation des caméras et la possibilité de leur commande à distance qui rendent possibles la production de perspectives nouvelles sur le match (caméras dans les filets, spidercam, etc.) ; l'incorporation de techniques venues du cinéma telles que le travelling ou la Steadycam permettant d'accompagner au plus proche et de façon dynamique la trajectoire des actions ; et, enfin, la numérisation des plateformes de ralentis qui permet d'accélérer considérablement l'accès à l'historique et de produire des ralentis dans des délais très restreints.

En somme, c'est toute la manière de se rapporter à la réalité qui évolue. Les phénomènes du match ne sont plus conçus comme relevant d'un donné déjà là et non-interrogeable, mais comme étant constitués par le travail des techniciens vidéo, c'est-à-dire par les mouvements de caméras et le montage. Les limites traditionnelles du visible sont repoussées, les caméras et les ralentis étant à la fois mobilisés pour rendre compte de l'action dans ses détails et pour donner accès à des espaces jusqu'alors tenus hors d'accès pour le spectateur (cf. Camus, 2017a). De plus, la temporalité de cette réalité du match télévisé évolue. Il ne s'agit plus d'une réalité évanescente, elle a désormais une épaisseur temporelle. La profusion des archives permet de rechercher et de mettre en images rétrospectivement des phénomènes, qui viennent ainsi rééclairer, donner un sens nouveau, à des actions qui leur sont postérieures. 
Ces pratiques de réalisation ont des conséquences importantes sur la manière dont sont représentés, perçus et décrits les actes d'arbitrage. Les images vidéo offrent de nouvelles possibilités de descriptions reconfigurant rétrospectivement les actions des joueurs et les jugements arbitraux portés sur elles. La production de ralentis, documentant l'action quelques secondes seulement après son avènement en direct, permet ainsi au téléspectateur de percevoir l'action sous des angles alternatifs, en ralentissant la séquence ou en glaçant l'image. Les phénomènes du jeu, vécus et accomplis de façon routinière dans la sphère de la coprésence, caractérisés par la logique du flux, l'évanescence, l'incertitude et la perspective située se retrouvent objectivés par l'image. Placé dans une position panoptique d'observateur transcendant, le téléspectateur se retrouve ainsi dans une position paradoxale : il a un accès au réel davantage documenté que l'arbitre qui a en charge de qualifier les faits. Cette asymétrie, qui peut être considérée comme étant à l'origine d'une attitude suspicieuse généralisée envers l'arbitrage, a vu dans l'adoption récente de l'assistance technologique de l'arbitrage (VAR) une forme d'aboutissement. Ce recours à la vidéo comme support pour juger le jeu accrédite l'idée selon laquelle la mise en images n'est pas une simple captation séparée du réel auquel elle donne un accès : en montrant la réalité, elle en transforme le sens et modifie la manière dont il est accompli in situ.

\section{La réalisation comme (re)construction du réel, un problème moral}

\section{La fidélité au réel mise en doute}

En raison de sa dimension rituelle et des évolutions de sa médiation, le football apparaît comme une activité de la vie sociale particulièrement propice à une réflexion sur la dimension morale des pratiques de représentation et des usages des technologies. Lorsqu'elle a été adressée comme telle, cette réflexion s'est inscrite dans le sillage des critiques traditionnelles des représentations médiatiques et a pris la forme d'une condamnation morale et d'une injonction à la réforme des pratiques de réalisation (Blociszewski, 2002). Derrière cette posture surplombante, il y a l'idée selon laquelle le match de football télévisé procèderait d'une « construction »- à la fois socio-historique et en temps réel - (Papa, 1998 : 281), idée qui se voit donc détournée en un problème moral : le match de football, à travers les procédures de réalisation qui viennent 
d'être évoquées, serait (re)construit en temps réel, brouillant la frontière entre réel et fiction (Blociszewski, 2007 : 39-40). À travers ces pratiques, le match vivant, celui des interactions en coprésence de l'espace du stade, courrait le risque d'être perdu au profit d'un match réélaboré par le montage, selon le regard subjectif que le réalisateur porte sur lui. Le téléspectateur se retrouverait ainsi privé de l'expérience originelle du direct télévisuel, du lien qu'il occasionne à l'événement en train de se faire, altérant ainsi la communion autour de ces cérémonies de confrontation au sens de Dayan et Katz (1992).

L'attitude, qui voit dans les procédures de mise en images le fait d'une reconstruction de la réalité, est caractéristique d'un certain type de constructivisme, qu'on pourrait qualifier de sceptique en raison de sa suspicion à l'égard des modes de perception des acteurs et la mise en doute de la fidélité de leurs représentations vis-à-vis de la réalité. Selon cette conception, trois critiques principales sont formulées à l'encontre des pratiques de réalisation.

La première porte sur le cadrage du match et son invisibilisation. Par l'insertion systématique de plan serrés et de ralentis, les réalisateurs restreignent la perception du téléspectateur et lui cachent une partie (ou la totalité) de ce qui se passe sur le terrain (Blociszewski, 2007 : 40-43). Cette restriction du cadre engendre une représentation centrée sur l'accomplissent individuel de l'action, masquant la coordination d'équipe. Elle contribue à amplifier le phénomène d'individualisation de la performance et de starification des joueurs (Diana, $2004: 25$ ), et biaise encore un peu plus la nature du jeu originel.

Une deuxième critique, qui découle de la précédente, concerne la modification de la temporalité du match (Blociszewski, 2001 : 6). Par l'insertion systématique de ralentis lors des périodes où le jeu est arrêté et par la multiplication des plans, le réalisateur accélère artificiellement le rythme du match. Il remet ainsi en cause l'expérience ordinaire du téléspectateur en occupant les « temps faibles » et en mobilisant son attention sur des phénomènes passés, contribuant ainsi à hybrider les temporalités de l'événement.

Une troisième critique est liée à l'usage des ralentis proprement dit et vise la transformation du match en « procès » ou en « enquête » (Blociszewski, 2001 : 8-10). L'arbitre, figure traditionnelle du jugement, est désormais au centre d'une enquête portant non pas seulement sur les faits de jeu mais sur la manière dont lui-même les a jugés. Cette enquête télévisuelle, qualifiée d'inquisitrice (Blociszewski, 2001 : 12 ; Diana, 2000), mobilise des outils dont l'arbitre ne dispose pas (du moins pas jusqu'à une date très récente) pour mettre en avant l'écart entre la réalité et les jugements arbitraux. En effet, la construction médiatique de cet écart est purement artificielle car les jugements arbitraux, en tant qu'ils sont les incarnations de la règle du jeu, peuvent 
être considérés comme des actes performatifs (Austin, 1970[1962]) : ils ne consistent pas seulement en une qualification des faits mais en leur factualisation ${ }^{3}$. Or, la réalisation, en interrogeant l'adéquation de ces jugements avec les faits, les traite comme de simples actes constatifs, c'est-à-dire comme des descriptions pouvant être vraies ou fausses et donc susceptibles d'être vérifiées.

\section{L'infidélité au réel : problèmes théoriques et méthodologiques}

Ces critiques, abondamment relayées par des médias spécialisés alternatifs comme les $\mathrm{Ca}$ hiers du Football ${ }^{4}$, des émissions de radio consacrées au football (en particulier l'After foot sur RMC) et parfois la presse généraliste (Le Monde ${ }^{5}$ Libération $\left.^{6}\right)$, s'accordent avec les vues de bon nombre d'amateurs éclairés de football s'inquiétant des évolutions de ce sport et, en particulier, de l'adoption du vidéo-arbitrage. Peu à peu, la mise en images du football émerge en tant que problème public alors que les difficultés techniques répétés liées à la VAR confirment les craintes anciennes de ses détracteurs ${ }^{7}$.

\footnotetext{
${ }^{3}$ En reprenant la classification d'Austin, accorder un but peut ainsi être conçu comme un illocutoire verdictif érigeant l'action du ballon qui roule au fond des filets en un événement comptable, rapportable aux règles du jeu, et ayant des conséquences irrémédiables pour la suite du jeu. Or interroger la véridicité de cet acte par le ralenti suppose de le transformer en un acte purement constatif, impliquant des conditions de vérité et donc une extériorité des phénomènes jugés.

${ }^{4}$ Voir notamment différentes interventions de J. Blociszewski, comme les trois volets « Football et télévision » en 2012 (http://www.cahiersdufootball.net/article-les-realisateurs-francais-hors-jeu-4503), l'article consacré aux plans serrés «L'invasion des visages » (2013) (http://www.cahiersdufootball.net/article-realisation-tele-football1-invasion-des-visages-5110), à la dissimulation du jeu par le montage « Pays-Bas-Espagne : et pendant ce temps, le ballon roulait » (2014) (http://www.cahiersdufootball.net/article-pays-bas-espagne-et-pendant-ce-temps-le-ballon-roulait-5338), la fictionalisation en 2019 « Non le match de football n'est pas une histoire» (http://www.cahiersdufootball.net/article-non-un-match-de-foot-n-est-pas-une-histoire-7291) ou des articles récurrents sur la question émanant d'autres auteurs, le dernier en date de 2020 étant « Réalisation télé : des matches à la tronçonneuse » (http://www.cahiersdufootball.net/article-realisation-tele-des-matches-a-la-tronconneuse-7409).

${ }^{5}$ Voir en particulier de nombreux articles dans le blog Le Monde de J. Latta Une balle dans le pied: « Coupe du monde : le football sous l'empire des images » (https://www.lemonde.fr/blog/latta/2014/06/20/coupe-du-mondele-football-sous-lempire-des-images/) ; mais également des articles parus dans la version papier tels que la tribune de J. Blociszewski en 2008 «Le football télévisé, opium du peuple?» (https://www.lemonde.fr/idees/article/2008/06/13/le-football-televise-opium-du-peuple-par-jacques-blociszewski_1057751_3232.html), ou O. Zilbertin « $\mathrm{Au}$ Brésil, le foot à l'école des réalisateurs européens" (https://www.lemonde.fr/culture/article/2014/06/23/au-bresil-le-foot-a-1-ecole-des-realisateurs-europeens_4443775_3246.html)

${ }^{6}$ Voir par exemple la tribune de J. Blociszewski en 2013 «PSG-OM sur Canal+: est-ce encore du foot?» (https://www.liberation.fr/sports/2013/02/26/psg-om-sur-canal-est-ce-encore-du-foot 884659) ou en 2014 «Mondial : les réalisateurs télé français ont moins le sens du collectif» (https://www.liberation.fr/sports/2014/06/11/les-realisateurs-francais-ont-moins-le-sens-du-collectif_1038324).

${ }^{7}$ Voir notamment le constat à propos du révélateur de hors-jeu de J. Latta, « One inch fails : la Premier League dans le piège du hors-jeu selon la VAR » en 2020 (https://www.lemonde.fr/blog/latta/2020/01/03/one-inch-failsla-premier-league-dans-le-piege-du-hors-jeu-selon-la-var/), faisant suite à G. Juan « La science confuse du révélateur » en 2014 (http://www.cahiersdufootball.net/article-la-science-confuse-du-revelateur-5222), quatre ans avant l'adoption généralisée de la VAR en 2018. Des articles divers sont consacrés à la question dans la presse,
} 
Ces controverses donnent notamment l'occasion d'une réflexion intellectuelle sur des processus médiatiques ordinairement « frappés du sceau de l'illégitimité culturelle » (Diana \& Lochard, $2004: 17$ ) et mettent en évidence l'importance des pratiques de mises en images en direct, en montrant qu'elles ne se réduisent pas à une simple captation transparente et passive du réel. Cependant, la dimension active du travail ordinaire de l'image est principalement présentée comme un problème, dont le remède pourrait être trouvé dans les recommandations de l'analyste qui, soucieux de « ne pas mettre le réel en danger », plaide pour un effacement de la subjectivité du réalisateur, un « respect du rythme réel du match », un renoncement à certains ralentis et l'observation d'une injonction morale au « respect » et à la « pudeur » (Blociszewski, $2002: 128)$.

Une telle conception, qui voit dans le match télévisé une (re)construction infidèle à sa réalité vécue sur le terrain, pose un certain nombre de problèmes, à la fois théorique et méthodologique, à rapprocher des critiques dont ont fait l'objet les conceptions constructivistes du social. L'une d'elles, mise en évidence par Woolgar et Pawluch (1985) sous l'expression d' " ontological gerrymandering » (généralement traduite par « charcutage ontologique »), vise l'opération de découpage du monde entre, d'une part, des objets et des pratiques dont le caractère socialement construit est révélé par l'analyste - en l'occurrence le match de football télévisé et, d'autre part, des régions du monde qui, affranchies d'un tel travail de révélation, se retrouvent naturalisées - le « match de football réel ».

Ce type de démarche pose notamment le problème de placer le chercheur apte à opérer un tel découpage dans une position de surplomb, faisant passer les pratiques de monstration correspondant à sa propre représentation de la réalité et à son propre système de valeurs, comme les pratiques les plus fidèles à la réalité. On voit donc que dans ces critiques, et à travers elles dans l'injonction morale adressée aux réalisateurs de respecter la réalité du match, se nichent des représentations du monde et des systèmes de valeurs divergents et, donc, l'expression d'une asymétrie entre la perspective du chercheur et la perspective des acteurs. La revendication d'une telle asymétrie dans l'accès à la « réalité » - en faveur de l'analyste - est d'autant plus problématique quand, comme c'est le cas ici, elle repose en fait sur une asymétrie de savoir-faire pratiques et de compréhension des enjeux interactionnels propres à l'activité, en faveur des enquêtés. Ainsi présentée, l'injonction à la fidélité à la réalité n'est en fait qu'une manière de

par exemple : «Football : l'arbitrage en mode vidéo gaga » (https://www.lemonde.fr/ligue-1/article/2017/12/18/football-1-arbitrage-en-mode-video-gaga_5231459_1616940.html) 
réintroduire insidieusement des valeurs et des représentations subjectives qui sont celles du chercheur, autrement dit une forme de morale exogène.

Un autre problème rencontré par ce type de perspective critique est de ramener les pratiques de représentation du réel à des phénomènes intentionnels. La réalisation apparaît ainsi comme la fait d'une volonté libre, affranchie à la fois de l'historicité des dynamiques structurelles télévisuelles et surtout des singularités contextuelles dans lesquelles prennent vie les pratiques. La démarche analytique consistant à adopter la perspective du téléspectateur empêche en effet l'analyste critique d'être attentif à la dimension contingente de la réalisation. Elle ne permet pas de voir dans les pratiques constitutives de la réalisation l'exercice de savoirs faires et de manières de voir incorporés et routiniers.

Un autre point qui découle du précédent est que, d'une façon finalement ironique, cette perspective critique individualise la performance de réalisation. Plutôt que de mettre en valeur la collaboration inhérente à ce type de dispositif et les compétences dont font preuve les différents techniciens (cadreurs, opérateurs-ralentis, script, etc.) pour représenter collectivement le monde, c'est la figure du réalisateur-auteur qui est mise en valeur et critiquée. Or, pourquoi ne pas voir là également un biais individualiste de notre époque dont le chercheur serait lui-même la victime?

S'ajoute enfin un dernier problème méthodologique : le constat d'un écart entre la « réalité brute » et ses représentations médiatiques se repose sur l'observation de ces mêmes représentations déclarées biaisées, qui sont pour le chercheur critique la seule ressource pour mener son analyse.

\section{La fidélité au réel comme « problème de membres »}

Une conception alternative réside dans l'analyse des pratiques de réalisation replacées dans leur environnement d'émergence, à savoir la régie en tant qu'elle est l'espace depuis lequel est coordonnée l'activité de l'ensemble des techniciens. Par contraste avec la perspective constructiviste exogène, cette approche consiste en une appréhension compréhensive et endogène des pratiques de réalisation et de la morale qu'elles exhibent. Plutôt que de postuler, par l'observation des programmes finaux, l'existence d'un écart entre le monde tel qu'il est et le monde tel 
qu'il est représenté à la télévision, cette démarche analytique consiste à interroger empiriquement les modalités par lesquelles les différents participants de la régie (réalisateur opérateursralentis et caméramans notamment), à travers leurs pratiques (sélection de plan, insertion de ralenti, mouvements de caméras), rendent manifestes leur compréhension de l'événement, et donc leur vision du monde. L'idée est donc de faire le lien entre les pratiques de perception située de l'événement par les techniciens derrière leurs écrans et les pratiques de mises en images. Ainsi conçue, la fidélité au réel n'est pas un problème purement spéculatif mais un " problème de membre » (Garfinkel, 1967), résolu en pratiques, de façon routinière, par les participants de la régie dans le cours de leur activité collective.

\section{Le lien réflexif entre les comptes rendus télévisuels et les phénomènes dont ils rendent compte}

Comme préalable à cette démarche, il y a le refus d'une position sceptique principielle caractérisée par la suspicion à l'égard des représentations des acteurs et fustigeant l'écart présumé entre ces représentations du monde et le « monde tel qu'il est » (dont on a vu qu'il n'était autre que le monde perçu selon le système de valeurs du chercheur). Il est ainsi possible d'appliquer une forme de principe de charité qui nous conduirait à admettre que les participants engagés dans une entreprise de mise en images d'un événement le donnent à voir fidèlement à ce qu'ils en perçoivent et comprennent. Évidemment cette perception, comme tout acte de perception, n'est pas une appréhension pure : elle est effectuée dans un environnement social et matériel donné, autrement dit dans un contexte qui confère son sens aux actions perçues.

Cette conception s'inspire de la notion, empruntée à l'ethnométhodologie, d'accountability (Garfinkel, 1967 : 1). Les membres du monde social, lorsqu'ils accomplissent une action de façon routinière et non-intentionnelle, rendent cette action compréhensible par les autres. Par là, ils manifestent leur appartenance à une communauté dans laquelle ces actions ont un sens donné et observable. De ce fait, les phénomènes se trouvent dotés de propriétés d'intelligibilité, de visibilité et d'ordre, autrement dit sont accountable. Ils offrent donc une prise aux membres de la société pour en produire des descriptions, des comptes rendus, qui peuvent prendre une pluralité de formes.

Un point important tient donc au fait que les accounts ne sont pas des descriptions pures, établies ex nihilo, d'une réalité. Ces descriptions sont des actions insérées dans le monde qu'elles décrivent et sont constitutives de cette réalité. Ainsi conçus, les accounts et les 
phénomènes du monde entretiennent un lien mutuellement configurant et dynamique, qualifié de « réflexif ». Cette réflexivité n'est pas un phénomène intentionnel ou mental, ni un privilège de l'analyste (Lynch, 2000) mais une propriété des pratiques. En tant qu'elles sont de part en part sociales et morales ${ }^{8}$, celles-ci sont à la fois douées d'une intelligibilité ordinaire et offrent ainsi des ressources (et des contraintes) pour produire des descriptions (ou des représentations) à leur sujet.

Le travail de l'analyste consiste donc à saisir cette réflexivité ordinaire des pratiques et, par un long travail ethnographique d'observation et d'acquisition des compétences des acteurs, à accéder à la perspective de sens commun des membres sur le monde. Il s'agit de comprendre comment, par les détails pratiques de leurs actions, les acteurs rendent manifeste une certaine compréhension de sens commun de la réalité et, ce faisant, rendent visible leur appartenance à un monde social dans lequel ces actions ont un sens donné.

Ce requisit pose un certain nombre de défis. Le premier est d'accéder à ces compétences qui, dans le cas des techniciens de l'audiovisuel, sont très perfectionnées, portent sur des détails, et dont la compréhension par l'analyste nécessite un long processus d'observation, d'acclimatation et d'apprentissage. C'est le rôle du travail ethnographique. Le deuxième défi est de parvenir à rendre compte des détails qui sont pertinents pour les participants par l'analyse. L'une des caractéristiques de ce type d'environnements tient à la grande vitesse avec laquelle les actions, constamment renouvelées, surgissent à travers les écrans, et les compétences dont font preuve les professionnels (et en premier lieu le réalisateur) pour s'ajuster à elles par l'improvisation et le recours à des routines organisationnelles incorporées. La saisie de cette dimension émergente de l'action est rendue possible par la mise en place d'un dispositif de captation vidéo, visant à documenter les interactions entre techniciens en régie et le mur d'écrans devant lequel le réalisateur coordonne les procédures de filmage et le montage. L'usage (par l'analyste) de la vidéo permet ainsi de saisir la dimension vécue de l'appréhension du monde par les acteurs, et donc d'appréhender les procédures réflexives par lesquelles ils mettent en images les événements. Un troisième temps consiste en la transcription de ces données pour rendre les analyses accessibles au lecteur ; celle-ci suit des conventions de transcriptions établies en analyse conversationnelle, permettant à la fois de rendre raison de la temporalité propre à l'accomplissement des actions et de la dimension visuelle des phénomènes (Mondada, 2018). De nombreux travaux de ce courant de recherche se sont intéressés aux espaces de travail outillés, où la vidéo est mobilisée pour percevoir et accomplir une activité collectivement (cf. Broth et al. (2014)

\footnotetext{
${ }^{8}$ Sur la dimension intrinsèquement morale de l'accountability, voir notamment Quéré (1987 :105-106).
} 
pour une introduction à ces travaux et Relieu (1999) pour une approche praxéologique de l'expérience télévisuelle).

Ce détour théorique et méthodologique a été rendu nécessaire par la volonté d'expliciter le lien réflexif qui existe entre les comptes rendus (télé)visuels d'une scène et l'accomplissement de l'action par les acteurs de cette même scène ou, pour le dire autrement, l'ajustement continu entre les pratiques de médiation et l'événement dont elles rendent compte (cf. Quéré, 2006). Conçu comme tel, il n'y a donc pas de raison d'opposer la conception réaliste de la réalisation du sport en direct (caractérisée par le recours majoritaire au plan large) et la conception documentaire (caractérisée par l'alternance rapide de plans larges et de plan serrés et l'insertion de ralentis pendant les pauses). Chacune correspond à des manières de voir et à des modes de représentation normés propres à un métier et à une époque, et chacune fournit un compte rendu de la réalité de l'événement conforme à des visions professionnelles incorporées (Goodwin, 1994).

Plutôt que de voir dans les pratiques récentes de réalisation des procédures dénaturant la réalité, il est possible de les présenter comme des manières alternatives de se rapporter au réel, et ainsi de porter une attention particulière aux efforts accomplis par les participants pour ajuster leurs actions (mouvements de caméras, montage, rembobinage de ralenti, etc.) aux trajectoires des actions qu'ils perçoivent. Pour le dire autrement, l'exigence morale à rendre compte du réel, au fondement du travail de réalisation du football, est accomplie sous des modalités différentes selon les situations dans lesquelles elle s'exerce. Ce que ces différences exhibent, ce sont donc autant des évolutions dans les modes de représentations, dans les attentes propres à une communauté professionnelle que, et c'est là un point essentiel, dans les modes de perception du monde.

\section{Perception de la réalité et production interactionnelle de son compte-rendu}

Trois exemples, correspondant à des reproches adressés à la conception documentaire de la réalisation, vont maintenant être développés pour illustrer les pratiques réflexives par lesquelles les professionnels de la régie rendent compte du match de football : la captation des scènes où un carton est brandi par l'arbitre (exemplifiant le problème de la temporalité) ; la mise en images des états mentaux (réinterrogeant le statut des phénomènes qu'on considère comme 
relevant de la réalité) ; et la production de l'objectivité du hors-jeu par le recours à un artefact visuel (posant le problème de l'autonomie de la réalité vis-à-vis de sa mise en images).

Le propos développé ici prend appui sur une méthodologie inspirée de l'ethnométhodologie et de l'analyse conversationnelle, consistant en l'observation vidéo-ethnographique en régie lors de la réalisation par les équipes de Canal + de matchs de football du championnat de France pendant deux saisons. Deux caméras ont été placées en régie de façon à saisir les interactions entre techniciens (réalisateur, scripte, opérateurs-ralentis, etc.) et les actions rendues visibles sur le mur d'écrans (comprenant à la fois les mouvements de chacun des 25 caméras, le rembobinage des 12 ralentis, et bien sûr les actions filmées elles-mêmes). Ce dispositif méthodologique vise à appréhender ces pratiques dans la temporalité de l'événement par l'analyse de séquences vidéo et leur transcription. Il permet ainsi d'étudier les modalités par lesquelles les participants font l'expérience de l'événement en temps réel et les savoir-faire pratiques qu'ils mettent en œuvre collectivement pour en rendre compte à un public distant.

\section{Le respect de la temporalité du match et l'occupation des temps faibles}

Lorsqu'une faute est sifflée par l'arbitre, le jeu est mis en pause pour un temps indéfini. Ces pauses sont nombreuses et peuvent être relativement longues. L'une des évolutions majeures mise en place par la conception documentaire de la réalisation réside dans l'exploitation de ces moments où le jeu est à l'arrêt comme des opportunités pour insérer des ralentis. L'action qui vient de se dérouler, dont le terme est un coup de sifflet la catégorisant comme faute, est alors revue sous un angle alternatif.

La production de ce type de ralenti se caractérise par sa rapidité d'exécution collective. Le coup de sifflet sert de ressource aux opérateurs-ralentis pour initier le rembobinage et produire une séquence du phénomène qui vient de se dérouler (voir Camus, 2017b pour un développement). Ces images peuvent ainsi être mobilisées par les commentateurs pour décrire rétrospectivement l'action et le jugement arbitral qui vient d'être rendu. Malgré sa dimension routinière et systématique, ce type de lancement de ralenti repose sur une observation attentive du cours du match et sur la capacité de l'ensemble des opérateurs (et en premier lieu du réalisateur) à anticiper la suite de la scène. En effet, c'est également lors de ces pauses que l'arbitre peut être amené à brandir un carton à l'un des joueurs. Or, les réalisateurs attachent de l'importance à montrer en direct tous les phénomènes relevant du jeu, ce qui inclut ces actes d'arbitrage 
incarnés. Pour pouvoir les rendre visibles, il est donc nécessaire d'anticiper le carton jaune et ainsi refuser les propositions de ralenti qui sont faites en régie après une faute. En retardant le lancement du ralenti pour montrer le carton jaune, le réalisateur rend ainsi manifeste le privilège qu'il accorde à la réalisation en direct des faits de jeu. Il s'agit de produire un ordre séquentiel des plans plaçant l'arbitre et le téléspectateur dans une symétrie de connaissance au moment où le jugement est rendu, le ralenti n'intervenant que dans un second temps, permettant ainsi de revoir non seulement l'action de la faute mais de la revoir en tant qu'elle a déjà été jugée par l'arbitre.

Anticiper un carton suppose et rend manifeste non seulement une connaissance de sens commun des règles du jeu - et la capacité de leur rapporter un événement singulier - mais aussi une compréhension de la scène se déroulant après que le jeu soit arrêté et de la manière dont les participants se rapportent eux-mêmes aux événements (par la trajectoire de leur corps, l'expression de douleur, les contestations, etc.). Tout cela n'est rendu possible que par l'organisation visuelle de la régie et par la multiplicité des caméras, des axes et des valeurs de plan qui rendent accessibles les détails de cette scène au réalisateur (cf. Camus, 2015 : 225-327 pour un développement).

\section{Où s'arrête la réalité ? Montrer les détails et capturer les états mentaux des joueurs}

La conception documentaire de la réalisation propose, comme on l'a dit, un grand nombre de plans serrés sur l'action. Ces insertions récurrentes ont été présentées de façon négative car considérées comme trop restrictives pour la perception du téléspectateur. L'un des usages des plans serrés est de rendre compte des états mentaux des joueurs. Il s'agit en effet de donner à voir des phénomènes a priori considérés comme relevant du privé, tels que des émotions (joie, colère, déception, tristesse, etc.) ou des sensations (douleur), comme des événements publics dont l'intelligibilité permet aux commentateurs et aux téléspectateurs de réinterpréter rétrospectivement une action passée 9 .

L'insertion de ces plans constitue un renversement important. Par le recours à ces plans donnant à voir la manière dont les joueurs éprouvent, vivent, de façon parfois charnelle, les

\footnotetext{
${ }^{9}$ De ce point de vue, ce qu'est «la réalité du match » gagne en complexité si on considère notamment les cas, fréquents, où l'affirmation de la réalité d'une faute (autrement dit le bienfondé d'un acte d'arbitrage) est mise en corrélation avec la réalité - potentiellement mise en doute - de la douleur exprimée par un joueur suite à un choc (Camus, 2019).
} 
événements dont ils sont les acteurs, le match n'est plus simplement une réalité objective dont la perspective surplombante rend compte. La réalisation permet ainsi de rendre visibles les appréhensions subjectives de l'événement. À travers les modalités par lesquelles les joueurs se rapportent à l'action qui précède, s'offre à l'analyse une forme de réflexivité élémentaire des participants sur le monde.

\section{L'enquête par l'image : l'objectivité comme production interactionnelle}

Une dernière critique est celle de la transformation du match en enquête et l'illusion d'objectivité qu'apportent les ralentis. Le révélateur de hors-jeu est de ce point de vue un objet particulièrement intéressant. Le hors-jeu est un phénomène qui, en tant qu'il est lié au positionnement dans l'espace des joueurs à un moment donné, pourrait paraître intrinsèquement objectif et ne pas relever de la subjectivité des techniciens. Le révélateur est une ligne artefactuelle délimitant par une image arrêtée l'espace du hors-jeu au moment où celui-ci doit être jugé par l'arbitre : celle-ci réifie le hors-jeu transformant un acte de jugement in situ, et en temps réel, en une réalité objective.

Or, une fois replacée dans son contexte d'émergence, cette objectivité ne peut être conçue comme étant toujours déjà là. Elle apparaît comme le fruit d'un ajustement continuel des journalistes et des techniciens à la scène qu'ils perçoivent et comme relevant d'un processus interactionnel. Le terme de ce processus tient dans l'évidence partagée du hors-jeu en tant que phénomène autonome et naturel, et l'oubli par les participants des efforts qui ont été nécessaires pour le faire accéder à sa visibilité et à son objectivité (Camus, à paraître).

Ainsi, plutôt que d'y voir une « inquisition » par l'image, le processus d'enquête inhérent à la réalisation pourrait être perçu de façon positive, selon une acception pragmatiste (Dewey, 2006[1938]), comme le processus local par lequel les participants essayent de produire de la connaissance à propos des situations dont ils font l'expérience. 


\section{Conclusion}

Le problème de la fidélité au réel est un problème qui se pose non seulement au chercheur traitant de son objet mais également à tout acteur du monde social qui, dans ses pratiques ordinaires et professionnelles, est amené à produire un compte-rendu à propos du monde. En raison de sa temporalité singulière, de ses évolutions techniques et de son importance rituelle, l'activité de médiatisation du football constitue un site particulièrement intéressant pour étudier les pratiques profanes par lesquelles les acteurs se rapportent à la réalité qu'ils perçoivent pour en produire un compte-rendu, qu'il soit visuel (réalisation) ou verbal (commentaire).

Dans cet article, deux conceptions de la morale sont envisagées en regard de ces pratiques. Une première, exogène, consiste à soutenir que le chercheur a une place privilégiée pour juger de la fidélité des représentations des acteurs vis-à-vis de la réalité. Cette conception peut être dite dualiste car elle soutient qu'il existe un écart entre la « réalité telle qu'elle est » et la réalité telle qu'elle est représentée. La seconde, défendue ici sous l'expression de moralité endogène, est une vision de la morale conçue comme incorporée dans les pratiques de perception et de représentation. Celle-ci est anti-dualiste et refuse l'affirmation a priori d'un hiatus entre les productions médiatiques et le monde qu'elles décrivent : les acteurs appartiennent au monde social qu'ils perçoivent et représentent selon les attentes morales et les normes propres à leur communauté (c'est-à-dire à leur époque, leur activité professionnelle, etc.) et les productions médiatiques sont donc constitutives de la réalité qu'elles donnent à voir. Cette morale est ainsi instanciée tacitement par les acteurs dans les situations singulières qu'ils rencontrent et parfois formulée explicitement lorsqu'ils s'interrogent collectivement sur le statut d'un phénomène. Ainsi conçue, la fidélité au réel apparaît non pas comme un problème spéculatif mais comme un problème pratique rencontré par les participants lorsqu'ils perçoivent et rendent compte du monde. Questionner la dimension morale inhérente aux pratiques de représentation passe donc par l'observation empirique du contexte dans lequel celle-ci s'exerce, plutôt que par la mise en doute a priori de l'adéquation à une réalité naturalisée (ou objectivée) par l'analyste.

Pour étudier la réalisation du football, l'article prône donc l'analyse située de sa dynamique de production par le recours à une vidéo-ethnographie des interactions entre techniciens en régie. Ni captation transparente ni pure reconstruction, le match télévisé apparaît ainsi comme le résultat des pratiques locales par lesquelles les professionnels font sens de la réalité qu'ils perçoivent à travers des écrans et la mettent en image par le montage, les mouvements de 
caméra ou le rembobinage. Le déplacement opéré ici entend donc rendre raison du processus interactionnel dynamique par lequel la réalité du match est constituée en mettant à jour des formes élémentaires, routinières et contingentes d'ajustement à l'événement en tant qu'il possède une intelligibilité propre (Quéré, 2006), ce que l'ethnométhodologie a thématisé sous le terme de réflexivité. 


\section{Bibliographie}

Austin John Langshaw (1970 [1962]), Quand dire, c'est faire, Paris, Le Seuil.

BIDEAUX François-Charles (2007 [1998]), « La révolution impossible », in Thierry Jousse (ed.), Le Goût de la télévision, Paris, INA, p. 604-607.

BlocisZewSKI Jacques (2001), «Le football télévisé victime du ralenti », Communication et langages, $\mathrm{n}^{\circ} 129$, p. 4-20.

BLOCISZEWSKI Jacques (2002), «France : quelle éthique pour le football télévisé ?», Les Cahiers du journalisme, $\mathrm{n}^{\circ} 11$, p. 120-133.

BlocisZEWSKI Jacques (2007), Le match de football télévisé, Rennes, Apogée.

BRoth Mathias, LAURIER Eric \& MONDADA Lorenza (2014), "Introducing Video at Work", in Mathias Broth, Eric Laurier \& Lorenza Mondada (eds.), Studies of Video Practices, New York, Routledge, p. 1-29.

CAILlOIS Roger (1967[1958]), Les jeux et les hommes, Paris, Gallimard.

CAmus Laurent (2015), Réaliser en direct. Une vidéo-ethnographie de la production interactionnelle du match de football télévisé depuis la régie, Thèse de doctorat en sociologie, Télécom ParisTech.

CAMUS Laurent (2017a), «Attendre le signal. Une analyse des pratiques de synchronisation de l'événement avec sa retransmission télévisée en direct », Réseaux, n²02-203, p. 341-372.

CAMUS Laurent (2017b), « La faute et son ralenti. Le cadrage temporel et visuel de l'action normée », Temporalités, 25, p. 1-20.

CAMUS Laurent (2019), "Seeing "real" pain or faking as an organizational issue in TV broadcasting of football matches", Présentation à IIEMCA Conference, Mannheim.

CAMUS Laurent (à paraître), « La production télévisuelle de l'objectivité : la révélation du hors-jeu en football », Réseaux.

DAYAN Daniel \& KATZ Elihu (1992), Media events: the live broadcasting of history, Cambridge, Harvard University Press.

DEWEY John (2006 [1938]), Logique : la théorie de l'enquête, Paris, PUF. 
DiANA Jean-François (2000), « Les enjeux du ralenti dans la représentation télévisuelle du football. Entre inquisition et réquisition », Cahiers de l'INSEP, 1(1), p. 255-269.

DiAnA Jean-François (2004), « Identité de l'image de sport », Médiamorphoses, n 11, p. 22 26.

DiANA Jean-François \& Lochard Guy (2004), « Les représentations médiatiques du sport », Médiamorphoses, ${ }^{\circ} 11$, p. 17-19.

GARFINKEL Harold (1967), Studies in Ethnomethodology, New Jersey, Prentice Hall.

GoodwIN Charles (1994), "Professional vision", American Anthropologist, 96 (3), p. 606633.

LYNCH Michael (2000), “Against reflexivity as an academic virtue and source of privileged knowledge", Theory, Culture \& Society, 17(3), p. 26-54.

MONDADA Lorenza (2018), "Multiple Temporalities of Language and Body in Interaction: Challenges for Transcribing Multimodality", Research on Language and Social Interaction, 51(1), p. 85-106.

PAPA Françoise (1998) «Les matchs sur le petit écran », Sociétés et représentations, n7, p. 281-294.

QUÉRÉ Louis (1987), « L’argument sociologique de Garfinkel », Réseaux, n²7, p. 97-136.

QUÉRÉ Louis (1991), «D'un modèle épistémologique de la communication à un modèle praxéologique », Réseaux, n46-47, p. 69-90.

QUÉRÉ Louis (2006), «Entre fait et sens, la dualité de l'événement », Réseaux, nº139, p. 183218.

RELIEU Marc (1999), « La réalisation et la réception du produit télévisuel comme accomplissements », in Jean-Paul Desgoutte (Ed.), La mise en scène du discours audiovisuel, Paris, L'Harmattan, p. 35-65.

TESSON Charles (2007 [1998]), «Filmer le football », in Thierry Jousse (ed.), Le Goût de la télévision, Paris, INA, p. 586-601.

Turowetz Jason J. \& MAYNARD Douglas M. (2010), "Morality in the social interactional and discursive world of everyday life", in Steven Hitlin \& Stephen Vaisey (eds.), Handbook of the Sociology of Morality, New York, Springer, p. 503-526. 
paru dans Télévision, 12, p. 103-120

WoOlgar Steven \& PAWLUCH Dorothy (1985), "Ontological Gerrymandering: The Anatomy of Social Problems Explanations”, Social Problems, 32 (3), p. 214-227. 